\title{
An artificial neural network approach for modelling the ward atmosphere in a medical unit
}

\author{
Mauro Lo Schiavo ${ }^{a}$, Barbara Prinari ${ }^{b, c, *}$, Jessica A. Gronski ${ }^{\mathrm{b}, \mathrm{d}}$, Angelo V. Serio ${ }^{\mathrm{e}, \mathrm{f}}$ \\ ${ }^{a}$ Univ di Roma "La Sapienza", Dip. di Metodi e Modelli Matematici per le Scienze Applicate, Roma, Italy \\ ${ }^{b}$ University of Colorado Colorado Springs, Department of Mathematics, Colorado Springs, CO \\ ${ }^{c}$ Univ del Salento, Dip.to di Matematica e Fisica "E. De Giorgi" and Sezione INFN, Lecce, Italy \\ ${ }^{d}$ University of Colorado Boulder, Department of Applied Mathematics, Boulder, CO \\ e Univ del Salento, Dipartimento di Storia Società e Studi sull'Uomo, Lecce, Italy \\ ${ }^{f}$ Dipartimento Salute Mentale ASL Brindisi - Italy
}

\begin{abstract}
Artificial neural networks (ANNs) have been developed, implemented and tested on the basis of a four-year-long experimental data set, with the aim of analyzing the performance and clinical outcome of an existing medical ward, and predicting the effects that possible readjustments and/or interventions on the structure may produce on it. Advantages of the ANN technique over more traditional mathematical models are twofold: on one hand, this approach deals quite naturally with a large number of parameters/variables, and also allows to identify those variables which do not play a crucial role in the system dynamics; on the other hand, the implemented ANN can be more easily used by a staff of non-mathematicians in the unit, as an on-site predictive tool. As such, the ANN model is particularly suitable for the case study. The predictions from the ANN technique are then compared and contrasted with those obtained from a generalized kinetic approach previously proposed and tested by the authors. The comparison on the two case periods shows the ANN predictions to be somewhat closer to the experimental values. However, the mean deviations and the analysis of the statistical coefficients over a span of multiple years suggest the kinetic model to be more reliable in the long run, i.e., its predictions can be considered as acceptable even on periods that are quite far away from the two case periods over which the many parameters of the model had been optimized. The approach under study, referring to paradigms and methods of physical and mathematical models integrated with psychosocial sciences, has good chances of gaining the attention of the scientific community in both areas, and hence of eventually obtaining wider diffusion and generalization.
\end{abstract}

${ }^{*}$ Corresponding author

Email addresses: mauro.loschiavo@sbai.uniroma1.it (Mauro Lo Schiavo), bprinari@uccs.edu (Barbara Prinari), jessica.gronski@colorado.edu (Jessica A. Gronski), enzo.serio.ps@gmail.com (Angelo V. Serio)

Preprint submitted to Math. Comp. Simul.

February 28, 2015

(C) 2015. This manuscript version is made available under the Elsevier user license http://www.elsevier.com/open-access/userlicense/1.0/ 


\section{Introduction}

Over the past decade there has been an increasing interest in mathematical models that describe and analyze complex structures and processes such as interacting systems of human beings. It is not infrequent that mathematical theories that are well-known in some research areas strongly contribute to the creation of new methods and perspectives even in fields remote to those that motivated their introduction. This is the case of the so-called artificial neural networks (ANNs), which in recent years have been applied as a statistical data modelling tool to atmospheric sciences [23, 45, 1, 30], energy systems [28, 26, 38, 16], experimental and clinical medicine $[6,43,3,48,31,44,20,19,4,37]$, medical diagnosis [29, 5, 7, 41], and a huge variety of other settings. An ANN is a mathematical/computational model inspired by the structure and functional aspects of biological neural networks. It consists of an interconnected group of artificial nodes, or neurons, arranged in three or more layers: the first layer consists of input data, and the last layer contains the outputs; in addition, there are one or more hidden layers that process the information and establish the correspondence from the inputs to the outputs. The ANN is characterized by the interconnection pattern between

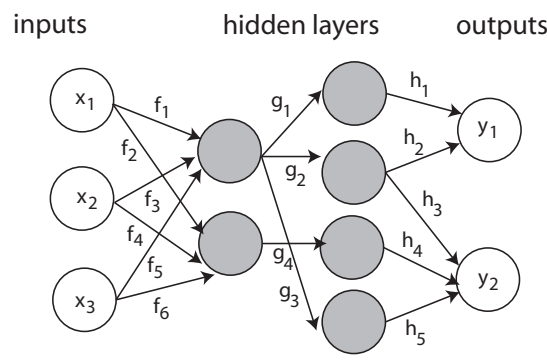

Figure 1: ANN schematics.

different layers of neurons, i.e., mathematically speaking, by the activation functions that convert each neuron's weighted input to its output. The network is an adaptive system that changes its structure based on external or internal information that flows through it during the learning process. In particular, in the so-called supervised learning, one uses a (sufficiently large) set of example pairs $(x, y)$ with $n$ inputs, $x=\left(x_{1}, \ldots, x_{n}\right)$ and $p$ outputs, $y=\left(y_{1}, \ldots, y_{p}\right)$, and the aim is to find the activation function in the allowed class that matches the examples. Mathematically, this is essentially often reduced to an optimization problem aiming at minimizing the absolute or averaged squared error between the network's output and the target value, $y$, over all the example pairs. The network training is achieved through a trial-and-error process, in which the network learns how to reproduce the correct outputs from the given inputs by adjusting the statistical weights on the connections between layers (Feed-forward/Back-propagation method) [15].

Motivation. In Refs. [32, 33] the authors have proposed a generalized kinetic model to describe the time evolution of a variable related to the quality and/or climate of a medical unit. The work was originally motivated by the analysis of experimental data collected for over 
10 years in an in-patient psychiatric unit (located in San Pietro Vernotico, Brindisi - Italy) under the direct responsibility of Dr. A.V. Serio, within the framework of a regional research project for monitoring and improving the quality of the service. The authors conceptualized the medical ward as a semi-closed system having two populations: patients and medical staff. The dynamics of the service, and the mutual relations among the individuals depend strictly on the "quality" that is offered (or that is perceived as being offered) by the service, i.e., on the social climate that is established. The experimental data are both quantitative and qualitative, and include: (i) monitoring a variable called Ward Atmosphere (atmosphere, for brevity), which refers generically to the social climate and is well-known in the specialized medical and psychosocial literature since the 1950s (see [53, 51, 46, 47, 39, 40, 49, 50]); (ii) presence of medical operators and staff; (iii) critical events (elopes, episodes of aggressiveness or violence, accidents); (iv) positive and negative events (visits, social activities, leaves of absence; troublesome admissions, such as for patients on compulsory sanitary treatment or at their first hospitalization); (v) flux data (input/output of patients). The atmosphere, in particular, has been measured 3 times a day since January 2001, according to a color code synthetically described in Table 1.

green: everything is fine in the ward and there are no negative emotions in the staff.

- blue: the behavior of one or more patients makes the staff feel uneasy.

yellow: the ward feels crowded, or there are patients in a more acute state, or behaving somewhat violently. The staff feels worried.

orange: the ward feels very crowded and one or more patients in critical conditions become problematic or aggressive, requiring some exceptional intervention. The staff feels on alert (fearful, powerless, etc).

red: it becomes necessary to restrain one or more patients, or to call for external help. The staff cannot withdraw.

Table 1: The color code for the Ward Atmosphere.

In order to allow numerical and statistical investigations on the collected data, the color code has been converted into the ordered set of five positive integers $\{2,4,6,8,10\}$, and hence on an equal-interval (ordinal Likert) scale, with 2 corresponding to green and 10 corresponding to red. Whether individual Likert items can be considered as interval-level data, or whether they should be considered merely ordered-categorical data is a subject of disagreement in the mathematical and psychosocial scientific communities. Such items could be regarded only as ordinal data, because, especially when using only five levels, one cannot assume that all pairs of adjacent levels are perceived as equidistant. On the other hand, to treat it merely as an ordered set without specifying a distance could imply losing important information. While extremely important when trying to model the dynamics of the system 
with a generalized kinetic approach (see [33]), this issue is much less relevant (if relevant at all) in the proposed ANN approach.

The ultimate goal of any mathematical investigation of the system is to predict, on a short-time scale, the possible outbreak of a crisis (orange/red ward atmosphere), and, on a long-time scale, the effects that specific planned or un-planned readjustments of the structure may produce on it and on patients and staff in terms of stress and satisfaction.

Identifying variables, parameters and events that control the dynamics, and thus being able to propose an effective mathematical model of the ward atmosphere and of its evolution is of the deepest importance from a social and health-care point of view (see, for instance, [52] for a recent study on how the ward atmosphere appears to mediate the associations between the physical and therapeutic characteristics of an inpatient ward and patient outcomes).

Methodology. In this work, we have developed, implemented and tested artificial neural networks to ultimately predict the ward atmosphere in the medical unit described above. The greatest advantage of using ANNs as a nonlinear statistical data modeling tool, as opposed to more "traditional" modelling approaches or simple statistical data analysis, lies in the fact that the network can learn from experimental data, i.e., it can be trained to infer the most effective modeling functions from observations. This feature is particularly useful in applications such as the one proposed in this paper, where the complexity of the data makes the design of such functions by hand impractical. At the same time, in order for the trained network to be robust, one needs a very large amount of experimental data (known input/output sets), which is a distinguished feature of the proposed application, as described below.

All the experimental data collected on a daily basis in the medical unit have been regarded as inputs/outputs of the network. The experimental outputs are vectors, whose values refer about the atmosphere measured in the ward during each of the three daily shifts. These values, in the reconstruction process, are used as a goal that the ANN must be able to simulate/reproduce. Therefore, only a small subset of them is used to initialize the ANN, and an even smaller subset to validate the most acceptable ones proposed by the initialization procedure. Specifically, for any given shift, the experimental output is identified with a 5component binary vector whose components represent the 5 classes, namely, the 5 colors for the atmosphere. Each experimental output vector then has a 1 in the component of the class corresponding to the atmosphere recorded by the staff in that shift, and four 0s in all other components. For instance, if the atmosphere recorded for a given shift is "blue" (i.e., 4 in our Likert ordered scale), the corresponding output vector is $y=(0,1,0,0,0)$. In this way, the simulated output computed by the ANN is a 5-component vector whose values represent the expected "probabilities" (in fact, only "likeliness", in that sometimes the outputs turn out to have small negative values) that are suggested by the ANN as corresponding to the given input, and hence may be compared with the experimentally measured ones.

The inputs, collectively a vector of 14 input data, are listed in Table 2 . It is important to point out that the intrinsic dynamics of the ward is based on three shifts per day, with the morning and afternoon shifts lasting 7 hours each (7am-2pm and 2pm-9pm, respectively), 


\begin{tabular}{|l|l|}
\hline INPUT & Description \\
\hline 1 & number of patients \\
2 & discharges \\
3 & new admissions \\
4 & troublesome admissions: patients on compulsory hospitalization \\
5 & troublesome admissions: patients at first hospitalization \\
6 & troublesome admissions: patients from out of state \\
7 & troublesome admissions: patients from out of county \\
8 & staff members present during the shift \\
9 & social workers \\
10 & total number of positive events \\
11 & negative/critical events: elopes \\
12 & negative/critical events: accidents \\
13 & negative/critical events: aggressions \\
14 & negative/critical events: restraints \\
\hline
\end{tabular}

Table 2: Components of the INPUT vector of the ANN

followed by the night shift which lasts 10 hours. There are, therefore, two different time scales in the system, with some experimental data being recorded on a daily basis, and others (among which, notably, the outputs) during each shift, hence three times a day. The first issue in the input/output setup has been to uniformize them all to a shift-based time scale, which has been dealt with as described below.

- The number of patients present in the medical unit is available per day, and it already accounts for the daily admissions and discharges; as an input value in the ANN, it is repeated for each of the three shifts during that day.

- Discharges and new admissions typically take place during the morning shift, therefore the corresponding input values are taken to be 0 for shifts 2 and 3 .

- Positive and negative events, as well as troublesome admissions, are also recorded on a daily basis, even though some of them, such as positive events and troublesome admissions, regularly occur only during the day (shifts 1 and 2), and negative events can occur suddenly and, obviously, somewhat unexpectedly, both during the day and at night. Nonetheless, the corresponding input values are repeated on each shift, assuming, based on experience, that their effects persist the entire day, if not even longer.

- Since social workers, if any, are only present in the morning shift, their presence as input is attributed to the first shift, and then taken to be 0 during shifts 2 and 3 .

We have then implemented and trained ANNs by means of the Neural Network (NN) Mathematica package, using the remarkably large experimental data set at our disposal (almost 
10,000 example pairs during between January 2001 and December 2004, and: 1) identified the input variables that play the most significant role in the dynamics; 2) determined the most suitable activation functions that yield reasonable convergence time and robustness; 3) used the trained ANN as a predictive tool on selected case periods, and compared its outcomes with the recorded outputs. An optimization of the network architecture has been carried out to identify the number of neurons, nodes in the hidden layers, network options, etc, in order to achieve the best balance between a satisfactory agreement during the training phase and the simplicity of the implementation. The predictions from the ANN technique have finally been contrasted and compared with the numerical simulations performed in [33], where a generalized kinetic approach was considered. Advantages of the ANN technique are twofold: on one hand, this approach deals quite naturally with a large number of parameters/variables, and also allows to identify those variables which are not relevant in the convergence of the training process (these are expected to play a marginal role in the dynamics and can therefore be ignored as inputs); on the other hand, the implemented ANN can be more easily used by a staff of non-mathematicians in the medical ward, as an on-site predictive tool.

The paper is organized as follows. In Sec. 2 we describe the mathematical model. In Sec. 3 we illustrate the outcome of the numerical simulations performed. In Sec. 4 we compare the outcome of the numerical simulations with the experimental data and briefly discuss the statistical significance of the model. Sec. 5 is devoted to some concluding remarks.

\section{Description of the specific model}

The ANN model which seemed more suitable to the specific problem is a Feed-forward network, which will be described in some detail below. Based on the previous investigation performed by means of a generalized kinetic approach [33], which clearly showed the system to be time-dependent, a plain Feed-forward network was anticipated to be too simplistic to model the system with some accuracy. And, as a matter of fact, the predictions of a plain Feed-forward network turned out to be quite unsatisfactory, way too "dull" to prove useful in order, for instance, to anticipate the possible outbreak of a crisis. In order to improve the description, we have then resorted to the use of "dynamic" neural networks, which have been implemented and tested on the system. A dynamic neural network is an ANN that utilizes networks such as Feed-forward networks or Radial Basis Function networks, and allows for the system to be time-dependent, i.e., for the output to depend not just on the corresponding input data, but also on the "history" of the system. Essentially, our dynamic neural networks model the system by constructing a Feed-forward ANN with suitably extended input vectors (regressors), where certain output values, 1 to 3 shifts before the one considered at each given time, are used as additional inputs. Since the dynamic ANNs implemented in this work are based upon Feed-forward networks, we will give below a description of the latter (in terms of training methods, options, etc), and then in Sec. 2.4 we will explain how the dynamic networks (and the corresponding regressors) are built upon them.

The process of data fitting uses the NeuralFit function of the NN package, in training both 
Feed-forward and dynamic networks. The object NeuralFit returns a list of two variables: (i) the trained network; (ii) the training record.

Each ANN has first been initialized (when possible) over a period of 14 days (i.e., 42 shifts) from Jan 07 to Jan 20, 2001. The initialization is performed in order to start the actual training with network parameters which are already somewhat "tamed" and hence likely to speed-up the convergence of the training process, instead of a random initialization which is the default option of the NN package. The initialized ANN is then trained over 126 days (378 shifts) from Jan 07 to May 12, 2001. Finally, the trained ANN is used to predict the atmosphere over two case periods, each lasting 28 days, carefully selected based on prototypical functioning of the medical service and of data collection procedures. The first case period runs from May 24 to June 20, 2001; the second one runs from Sep 13 to Oct 10, 2001. In these runs the trained ANN is an actual predicting tool, since only input data and, for the dynamic networks, outputs from (up to) a day (three shifts) before the one considered (see Sec. 2.4) are provided to the network, and the predicted outputs are compared with the experimentally measured ones to analyze the performance of the trained ANN. "Validated" ANNs have then been constructed, where one of the two case periods is used as the "validation" set, and the outputs are then predicted on the other case period. Each time, after the network has been trained, the validation set is used to calculate an error, and the training is stopped when the error on the validation set reaches a minimum. In other words, the error is minimized with respect to the training data and the obtained parameter estimates for the network are the ones that gave the best performance at some intermediate iteration during the training on validation data. The validation aims at preventing network overtraining, which can lessen its performance.

In order to double-check the results provided by the NN package in the training, each ANN has been initialized and then trained in two different ways: (i) through the ARXNet instruction in the package, which builds the Feed-forward network automatically; (ii) "manually", i.e., by means of a code implemented by the authors to perform the same operations. Similarly, in the prediction/validation processes the ANNs have been applied by means of the NetPredict instruction and, separately, manually. It is also worth mentioning that for all the numerical experiments the training has been repeated over the same period, yielding the same results.

\subsection{Feed-forward neural networks (FFNNs)}

A single-output FFNN is an artificial neural network that consists of hidden layers of neurons, with each neuron performing a weighted summation of inputs $\left\{x_{j}\right\}_{j=1}^{n}$, and then passing these weighted summations through a nonlinear activation function (typically, a sigmoid: $\sigma(x)=1 /\left(1+e^{-x}\right)$, meant to saturate the outputs to values between 0 and 1 corresponding to each output):

$$
\sigma\left(\sum_{j=1}^{n} w_{j} x_{j}+b_{j}\right) .
$$

The output layer then performs another weighted summation on the output of the neurons in the hidden layer, and provides our real-values predicted output, $y_{p}$. More specifically, the 
output corresponding to the given set of input data $\left\{x_{j}\right\}_{j=1}^{n}$ is given by

$$
y_{p}(\theta)=g(\theta, x)=\sum_{i=1}^{m} w_{i} \sigma\left(\sum_{j=1}^{n} w_{i, j} x_{j}+b_{j, i}\right)+b,
$$

where $m$ denotes the number of neurons contained in the hidden layer, $n$ is the number of inputs, and the set $\left\{w_{i, j}, b_{j, i}, w_{i}, b\right\}$ contains all parameters of the network, which are, for ease of representation, expressed by $\theta$ in $g(\theta, x)$. The parameters $w_{i, j}, i=1, \ldots, n, j=1, \ldots, m$ and $\left\{w_{i}\right\}_{i=1}^{n}$ are referred to as "weights", while $b_{i, j}, i=1, \ldots, n, j=1, \ldots, m$ and $b$ are the so-called bias parameters. In this work, ANNs with $m=0,4,7$ neurons in the hidden layer have been considered.

Once a set of inputs/outputs data are provided, the network undergoes a training process where the statistical weights and bias parameters are iteratively adjusted, through a back-propagation algorithm, so that the outputs provided by (2.1) are within a prescribed tolerance from the "real" outputs of the system.

\subsection{FFNN training methods and options}

To consider a network successfully trained, the (suitably defined) error calculated at each iteration must be sufficiently small, within some precision estimate typically provided by the user. In particular, for a single-output ANN the Mean-Squared Error (MSE) is defined by:

$$
V_{N}(\theta)=\frac{1}{N} \sum_{i=1}^{N}\left(y_{i}-g\left(\theta, x_{i}\right)\right)^{2}
$$

where $N$ is the number of data points provided. Correspondingly, a Mean-Squared Error Function is defined as:

$$
\operatorname{msef}(\epsilon)=\frac{\epsilon^{T} \epsilon}{l_{\epsilon}}=V_{N}(\theta)
$$

where $\epsilon=y-y_{p}$, i.e., the difference between the provided and generated output data members, also referred to as the "prediction" error; $l_{\epsilon}$ denotes the length of $\epsilon$. The generalization of the above definitions to multi-output ANNs, where the output is a $p$-component vector, is straightforward.

Each set of parameters $\theta$ is adjusted at every iteration in such a way that the parameters are estimated by the minimization of $V_{N}(\theta)$. Specifically, the parameters are adjusted at each iteration as such:

$$
\theta^{i+1}=\theta^{i}-\mu R \nabla_{\theta} V_{N}(\theta),
$$

where $R$ is the direction matrix, and $\mu$ is a predefined scalar controlling the size of $\theta$ or the step-length. $R$ is determined in the training method chosen for or by the network. Starting with $\theta^{0}$, the network iteratively attempts to decrease the MSE by incrementally updating $\theta$ along the negative gradient of the MSE.

Among the various training methods, the most common ones, i.e., the Levenberg-Marquardt (LM) method and the Gauss-Newton (GN) method, have been used in this work. Comparison of the performances of both training methods on both case periods has indicated the 
LM method as the most efficient of the two for the case study. Also, several numerical experiments have been performed with different FFNN options used in the training, in order to identify the most suitable options for the model. The key options resulted to be regularization and separability, which are briefly discussed below.

Regularization. When setting the option Regularization to a positive value, the criterion function to be minimized by the network in the training becomes:

$$
W_{N}^{\delta}(\theta)=V_{N}(\theta)+\delta \theta^{T} \theta
$$

where $\delta$ is the regularization parameter specified. $\delta \theta^{T} \theta$ is referred to as the regularization term, which acts as a spring pulling less important parameters towards the origin and only marginally influencing those parameters important to $V_{N}(\theta)$. This process reduces the number of effective parameters. The choice of the regularization parameter $\delta$ is by trial and error.

Separability. The separable algorithm fits the linear parameters in each step of the iterative training with the least squares algorithm. Separable training can be used when a neural network is linear in some of the parameters, since it gives a numerically better-conditioned minimization problem that is easier for the network to solve. The primary focus of the separable algorithm is to minimize the criterion in the direction of the linear parameters in each iteration so that the iterative training 'follows' down the valley to a minimum.

Note that for neural networks, flexibility is specified by the number of hidden neurons. Deciding on the correct amount of flexibility is therefore a tradeoff between two primary sources of misfit. This is referred to as the bias-variance tradeoff. The are three ways to handle this occurrence:

- Try different neural networks with various numbers of hidden neurons and test those networks on validation data; choose the best network.

- Specify a regularization parameter greater than 0 . This leads the network to minimize the regularized performance index as opposed to the original MSE. This type of regularization is usually referred to as weight decay in connection with neural networks.

- By submitting validation data, one applies stopped search. In this case, the MSE is minimized with respect to the training data and the obtained parameter estimate is the one that gave the best performance at some intermediate iteration during the training on validation data.

All of the above possibilities and combinations thereof have been explored in this work, and the predictions of the best performing resulting ANNs will be illustrated in Sec. 3 . 


\subsection{Dynamic neural networks}

A dynamic neural network is an artificial neural network that utilizes either FeedForward or Radial Basis Function networks and allows for the system to be time dependent, by combining FeedForward ANNs with time dependent input vectors called regressors. Generally speaking, the regressor is specified by three sets of indices: $n_{a}, n_{b}, n_{k}$, where $n_{a}$ refers to the number of lagged output values, $n_{b}$ refers to the number of lagged input values, and $n_{k}$ refers to the input delay relative to the output. For Single-Input, Single-Output (SISO) systems, $n_{a}, n_{b}, n_{k}$ are given as three positive integers. For Multi-Input, Multi-Output (MIMO) systems, each one of them is a list with one component for each input or output, i.e., each individual lagged signal value is a vector of appropriate length. This means that instead of each input data set $x(t)=\left(x_{1}(t), \cdots, x_{14}(t)\right)$, a "regressor" is used, conveniently constructed on account of a set $n_{a}, n_{b}, n_{k}$ in such a way that the input is $n_{b}$-shifted, $n_{a}$-augmented, and then properly $n_{k}$-paired with the output set $y(t)$. In our specific problem, with 5 output values, $n_{a}=\left\{n_{a}^{1}, \cdots, n_{a}^{5}\right\}$ and for each $t$, the regressor $t$-set is constructed as follows:

$$
\begin{aligned}
x(t)= & \left(y_{1}(t-1), \ldots, y_{1}\left(t-n_{a}^{1}\right), y_{2}(t-1), \ldots, y_{2}\left(t-n_{a}^{2}\right), \cdots, y_{5}(t-1), \ldots, y_{5}\left(t-n_{a}^{5}\right),\right. \\
& \left.x_{1}\left(t-n_{k}^{1}\right), \ldots, x_{1}\left(t-n_{k}^{1}-n_{b}^{1}+1\right), \ldots, x_{14}\left(t-n_{k}^{14}\right), \ldots, x_{14}\left(t-n_{k}^{14}-n_{b}^{14}+1\right)\right),
\end{aligned}
$$

and paired with the $y\left(t+\max \left(n_{b}+n_{k}\right)-1\right)$. As mentioned above, $y=\left(y_{1}, \cdots, y_{5}\right)$ denote the outputs, and $x=\left(x_{1}, \cdots, x_{14}\right)$ denote the user provided inputs. In particular, we have then chosen the 5 -plets and the two 14 -plets of values $\left(n_{a}, n_{b}, n_{k}\right)$ as follows: $n_{b} \equiv(1, \cdots, 1)$, $n_{k} \equiv(0, \cdots, 0)$ and two different logics have been adopted for the lagged outputs: (i) $n_{a}^{j}=1$ for $j=1, \cdots, 5$, which corresponds to simply appending as an additional input the information on the atmosphere recorded during the shift prior to the one considered; (ii) $n_{a}^{j}=j-1$ for $j=1, \cdots, 5$, where the output information provided as additional input to the network goes from 0 to 4 shifts back, the further back the more negative the atmosphere. It should be noted that in all dynamic ANNs the actual input/output pairs considered in the training are $n-\max _{j} n_{a}^{j}$, instead of $n$, as the pairs with $i=1, \cdots, \max _{j} n_{a}^{j}$ would require using output information that might not be available to the user.

Default predictions are one-step predictions, called "horizon" 1 (or $h=1$, for shortness), and denoted by $\hat{y}(t \mid t-1)$, i.e., predictions based on measured outputs up to time $t-1$. This kind of prediction is similar to the mechanism that might be actually used in the ward. It is worth stressing the difference between setting the horizon $h=1$ in the model, and setting the lagged output $n_{a}^{j}=1$ in the regressor, which corresponds to using the measured output values at the time previous time-step as additional inputs in the ANN. It is obviously interesting to check if a model is capable of performing predictions several time-steps ahead, that is, to increase the so-called prediction horizon. In this work, a three-step ahead (horizon 3 , or $h=3$ ) prediction $\hat{y}(t \mid t-3)$, where only past values up to $t-3$ are given, has been considered. This is accomplished by using the estimate $\hat{y}(t \mid t-3)$ in place of the missing $y(t-1), y(t-2)$ values for the normal one-step prediction. If the prediction horizon is infinity, it is said that the model is being used in simulation. Then, measured outputs are not used at all and all outputs in the regressor are replaced by model outputs. 


\section{Numerical simulations}

A large number of numerical simulations has been carried out, using dynamic FFNN, with various options, and their predictions validated over the two case periods May 24-June 20, 2001 and Sep 13-Oct 10, 2001.

Output likeliness. In the numerical simulations the trained ANNs return the "likeliness" of outputs for each input in the time series considered. Typical ANN outcomes for several choices of the network options are illustrated in Fig. 2, where the 5 colored splines, plotted as functions of the shifts, represent the likeliness of the corresponding outputs: e.g., the green spline gives the likeliness of an output/atmosphere corresponding to "green" or '2' (cf Table 1), the blue spline gives the likeliness of an output/atmosphere corresponding to "blue" or " 4 , and so on. It should be pointed out that, although the 5 values for the likeliness are normalized to sum to 1 , strictly speaking this likeliness cannot be interpreted as a probability, as in some cases the ANN returns small negative values for it. While a cut-off could be introduced to avoid this problem, in this work this issue has turned out to be of no practical importance in the further conversion of the output likeliness into suitable averages that represent the estimate for the ward atmosphere on each shift.

As explained in Sec. 2, validated ANNs have also been constructed, where one of the two case periods is used as validation set, and the outputs is predicted on the other case period. In the validated ANN, the error is minimized with respect to the training data and the obtained parameter estimates for the network are the ones that gave the best performance at some intermediate iteration during the training on validation data.

Moreover, in all the numerical simulations, predictions over the two case periods have been tested both as one-step predictions, and with a prediction horizon equal to 3 .
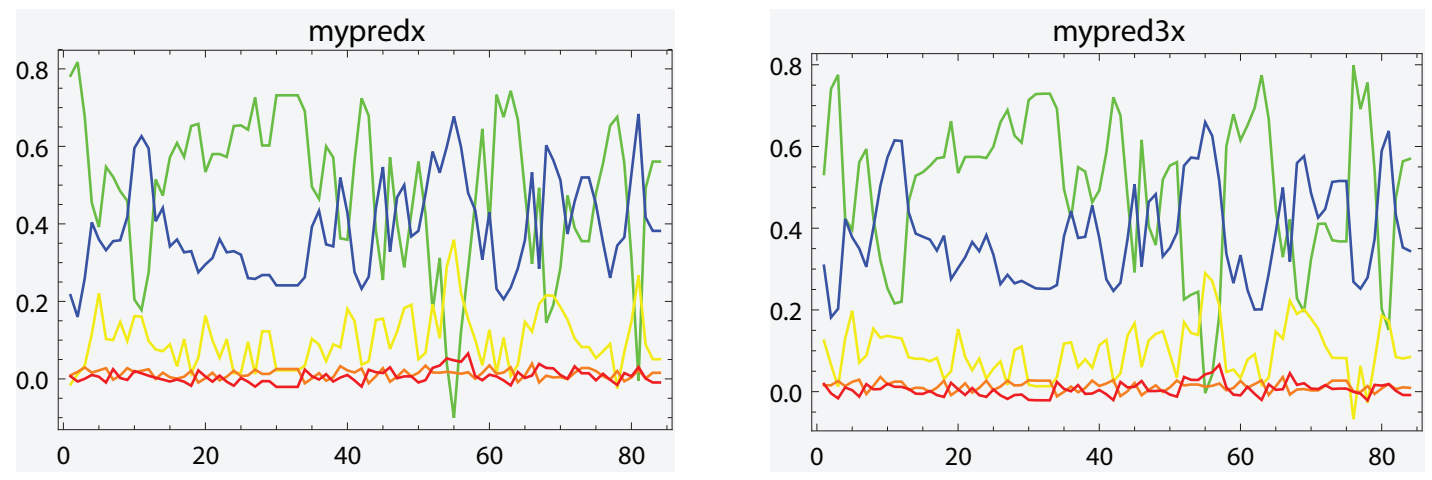

Figure 2: Dynamic FFNN prediction over the first case period (May 24 to June 20, 2001) with the second case period used as validation. Output likeliness for each class on the vertical axis are plotted vs each shift in the period. FFNN options: 7 neurons; no regularization; separable; Levenberg-Marquardt training. (Left) with horizon=1; (Right) with horizon=3. 
Predicted atmosphere. In order to compare the predictions of the model with the experimental data, it is necessary to convert the output of the numerical simulations, i.e., the likeliness discussed above, into a single sequence of integer values in the set $\{2,4,6,8,10\}$ that represent the estimate for the ward atmosphere on each shift. A similar problem had presented itself in the kinetic approach proposed in [33], whose outputs were generated as five probability density functions over a suitably defined state variable for the two populations of patients and staff in the given period. In this respect, the ANN approach is simpler to deal with in that there is no distinction in the ward population in patients and staff, the system is considered as a whole and the likeliness of a given output atmosphere is given as a single output vector. Conversely, some of the quantities identified in [33] as indicators/averages, whose discretizations could be reasonably compared with the experimental data for the atmosphere, seems now to be unjustified. For instance, the value $U_{c}=\alpha U_{1}^{c}+(1-\alpha) U_{2}^{c}$ introduced in [33] as a suitable weighted average of "critical" mean activities for operators and patients may be meaningful only if they are treated as two separate populations, which is not the case in the ANN approach presented here. As a consequence, the indicators/averages based on $U_{c}$ are not particularly useful in the present setting. Nonetheless, other averages can be applied with only slight adjustments to the present model, and in particular following the same logics as in [33], in this work the predicted atmosphere in a given shift has been computed from the likeliness by means of the following algorithm:

$$
U=\mathcal{N} \sum_{j=1}^{5} u_{j} y_{p}(j)
$$

where $y_{p}(j)$ is the likeliness of class $j$ provided as the output of the ANN in the shift considered, $\left(u_{1}, \cdots, u_{5}\right)=(0.1,0.3,0.5,0.7,0.9)$ are the "weights" attributed to each class, and $\mathcal{N}$ is the number of patients and staff members present in the ward during that shift. The weights are the same used in [33] to compute similar averages, and they have been chosen in order to emphasize the rarity of values 8 and 10 for the atmosphere. Next, following [33], in order to compare the preceding value $U$ to the experimental data, which are Likert-ordered as explained in Sec. 1, a discretization of $U$ has been considered, with threshold values chosen as those that appeared to provide good approximations to the experimental values for the atmosphere. Specifically, based on the variable $U$, the following estimate for the atmosphere has been considered:

$$
\begin{array}{ll}
U^{*}=2 & \text { if } 0.0 \leq U<1.7, \\
U^{*}=4 & \text { if } 1.7 \leq U<2.5, \\
U^{*}=6 & \text { if } 2.5 \leq U<5.0, \\
U^{*}=8 & \text { if } 5.0 \leq U<6.0, \\
U^{*}=10 & \text { if } U \geq 6.0 .
\end{array}
$$

Clearly, quantitative comparisons with experimental data are severely biased by the choice of the thresholds in the discretization, and only a careful a posteriori test may provide the best choice for their values. Nonetheless, if one is mainly interested in a qualitative estimate, then small differences in such choices may be ignored. As a matter of fact, a qualitative 
comparison between the estimates and the experimental values for the atmosphere turned out to be easier and more informative by looking directly at the "continuous" average, so only $U$ are shown in Figs. 3 and 4 below for the two case periods.

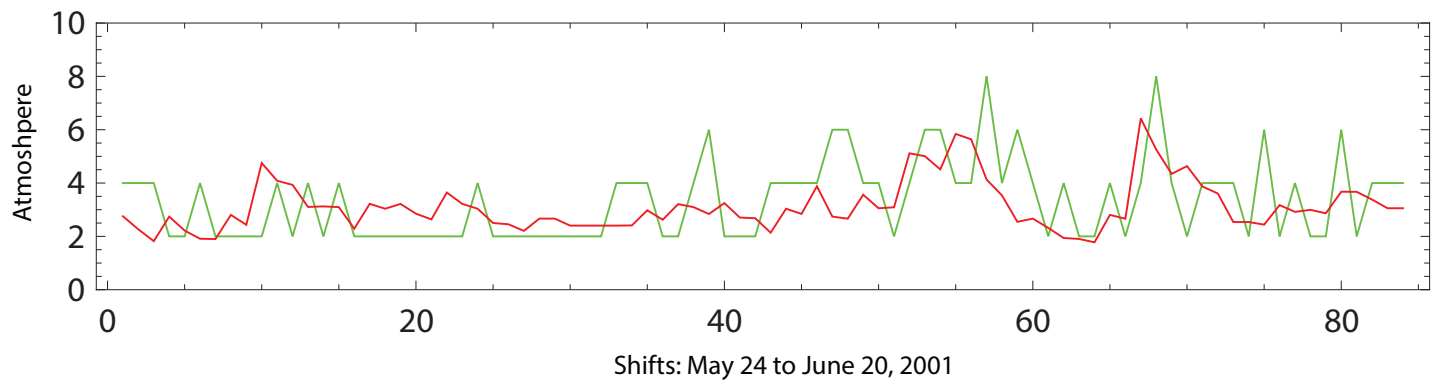

Figure 3: Atmosphere during the first case period, May 24 to June 20, 2001. Green: Experimental data; Red: prediction of $U$ from the ANN in Fig. 2 with horizon=1, computed according to (3.1).

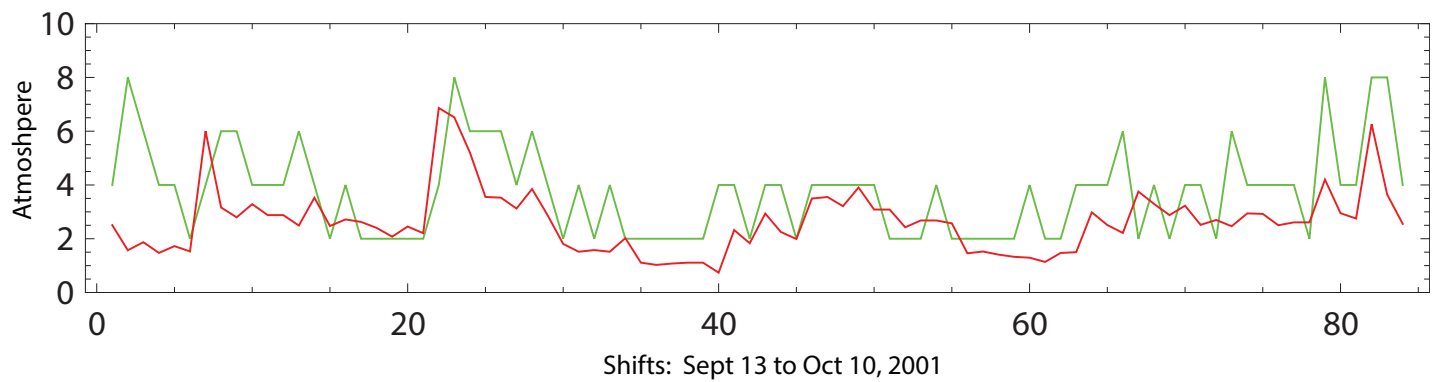

Figure 4: Atmosphere during the second case period, Sep 13 to Oct 10, 2001. Green: Experimental data; Red: prediction of $U$ from the ANN in Fig. 2 with horizon=1, computed according to (3.1).

\section{Comparison with experimental data and statistical significance}

In order to investigate the statistical significance of the model and to obtain a quantitative comparison, some global indicator has to be identified. To this aim, we first compute some straightforward mean square deviations between experimental and estimated values for the atmosphere. More specifically, the entire period of the analysis (January 2001 - December 2004) has been divided into 52 periods of 28 days each. Then, we denote by $v_{j}, j=1, \ldots, N$ the experimental value for the atmosphere at each shift $j$ of the period, and by $p_{j}$ the ANNsimulated value, according to the algorithm listed above for $U$. Hence, $N=84$ is the total number of evaluations per each of the periods under consideration. We introduce square 


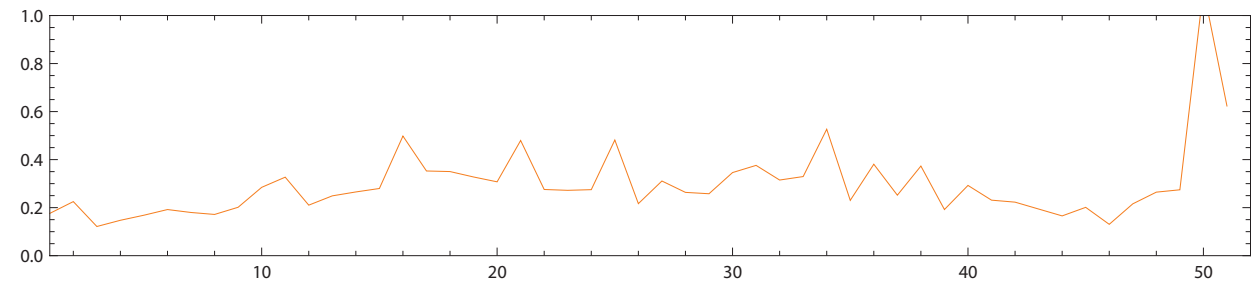

Figure 5: Mean deviation $S d / S v$ for the ANN outputs between January 2001 and December 2004 computed using the ANN in Fig. 2 with horizon=1.

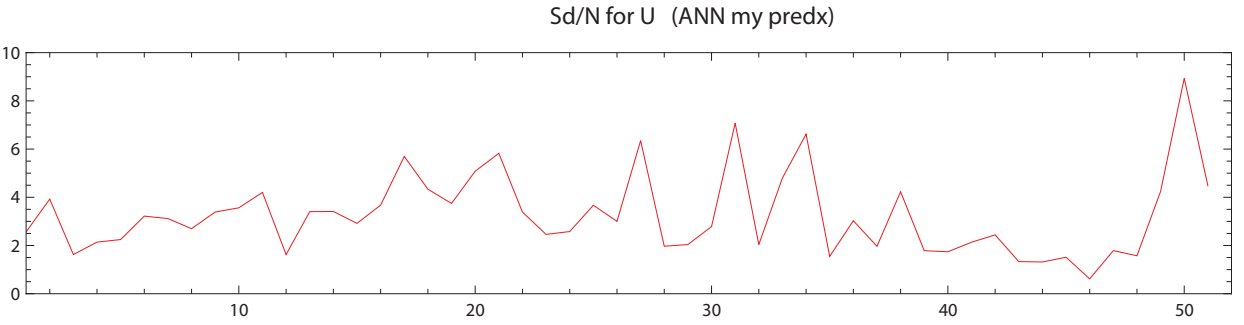

Figure 6: Mean deviation $S d / N$ for the ANN outputs between January 2001 and December 2004 computed using the ANN in Fig. 2 with horizon=1.

deviations:

$$
S d=\sum_{j=1}^{N}\left(v_{j}-p_{j}\right)^{2},
$$

as well as the sums

$$
S v=\sum_{j=1}^{N} v_{j}^{2}
$$

The values of the deviations $S d / N$ and $S d / S v$ are given by the polygonal lines in Figs. 5 and 6 , where each point corresponds to one of the aforementioned 52 periods.

Clearly, for both mean deviations illustrated in Figs. 5 and 6 there cannot be a "reference" graph; since they represent deviations of the predicted atmospheres with respect to the experimental values, they are obviously expected to be as small and "flat" as possible.

An alternative and somehow more sophisticated attempt of statistical analysis has been performed, in order to compare the experimental values for the atmosphere with the estimates from the numerical simulations by means of indicators that are well-known in the specialized psychological and social literature. In particular, the values of the Pearson and Spearman correlation coefficients between the experimental data and two of the estimates above have been evaluated and given in the following figures. As before, each point in Fig. 7-8 corresponds to one of the aforementioned 52 periods.

Unlike Figs. 5-6, in Figs. 7-8, obviously, the agreement is expected to be better the larger the values of the correlation coefficients. Even though it is questionable whether one can 


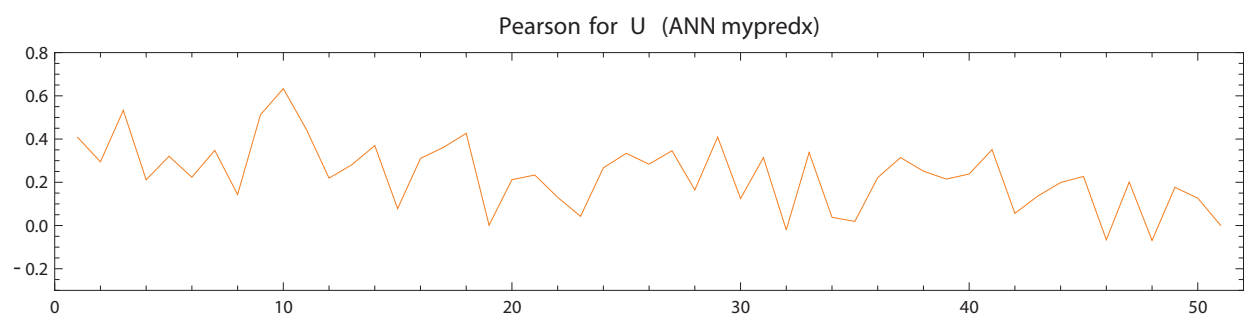

Figure 7: Pearson's correlation coefficient for the values of the atmosphere predicting using the ANN approach.

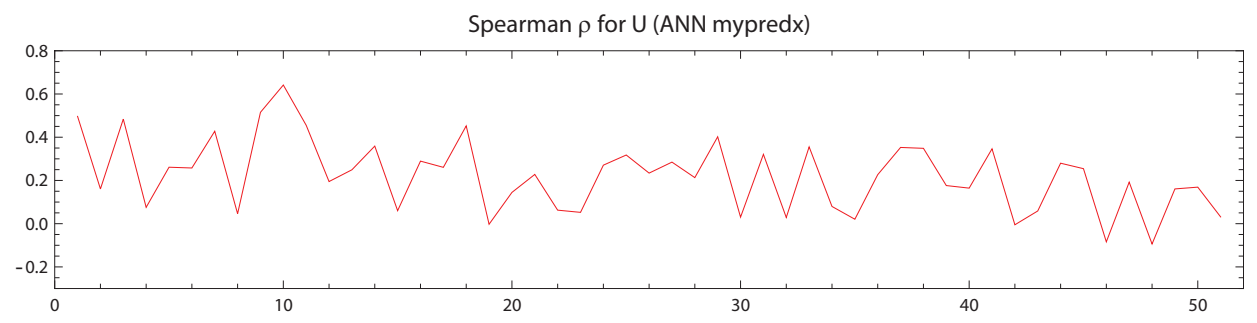

Figure 8: Spearman $\rho$ correlation coefficient for the values of the atmosphere predicting using the ANN approach.

use linear statistics to compare these data, we observe that for most periods the "small deviations' and 'large correlation' approaches tend to agree.

Also, it should be pointed out that for most of the periods that we analyzed, the values obtained for the correlation coefficients, from the point of view of psychosocial statistics are considered to indicate a remarkably good correlation among the data. It is also clear that both correlations decrease over time, and more so towards the end of the time frame considered in the analysis (2001-2004).

\section{Comparison with the kinetic model}

Finally, we propose here a comparison between the predictions of the ANN approach illustrated in the previous sections and the ones obtained by the authors in [33], where a (generalized) kinetic model had been developed with the aim of analyzing the ward atmosphere of the same medical unit. Generalized kinetic models transfer the methodology developed for systems of a great number of interacting particles (such as Boltzmann and Vlasov equations, with direct interactions among the particles or mean field terms and external forces [17]) to various other fields of research, such as traffic dynamics (see, for instance, the review paper [12]), cellular dynamics (see, among others, [18, 8, 9, 13, 12]), social and population dynamics $[27,14,34,12,24]$ and biological systems in general [10, 12]. Various models of the classical mathematical kinetic theory have been investigated and generalized in several contexts, $[22,2]$. The interested reader can find thorough reviews of the theory 
and applications of generalized kinetic models in [25, 11, 12]. Under certain simplifying assumptions, the kinetic model in [33] was ultimately reduced to a sequence of correlated initial value problems for a system of 10 coupled, nonlinear, nonlocal ordinary differential equations for the probability variables. The equations depended on a (fairly large) set of physical parameters that refer, at a "mesoscopic" scale, about the nature and frequency of the direct interactions among the actors (patients and staff) in the medical unit, as well as the effect of external positive or negative events, workload, and social terms (which specify how the individuals are affected by the overall ward state). The tuning of model parameters so as to best fit the experimental data had been performed over the same two case periods considered in this work.

First of all, it is instructive to look at the predictions of the two models for the atmosphere over the two case periods. Fig. 9 shows a comparison of predicted vs experimental values for the atmosphere over the two case periods, as obtained from the ANN validated over the second period and considered in the previous sections, and from the kinetic approach.
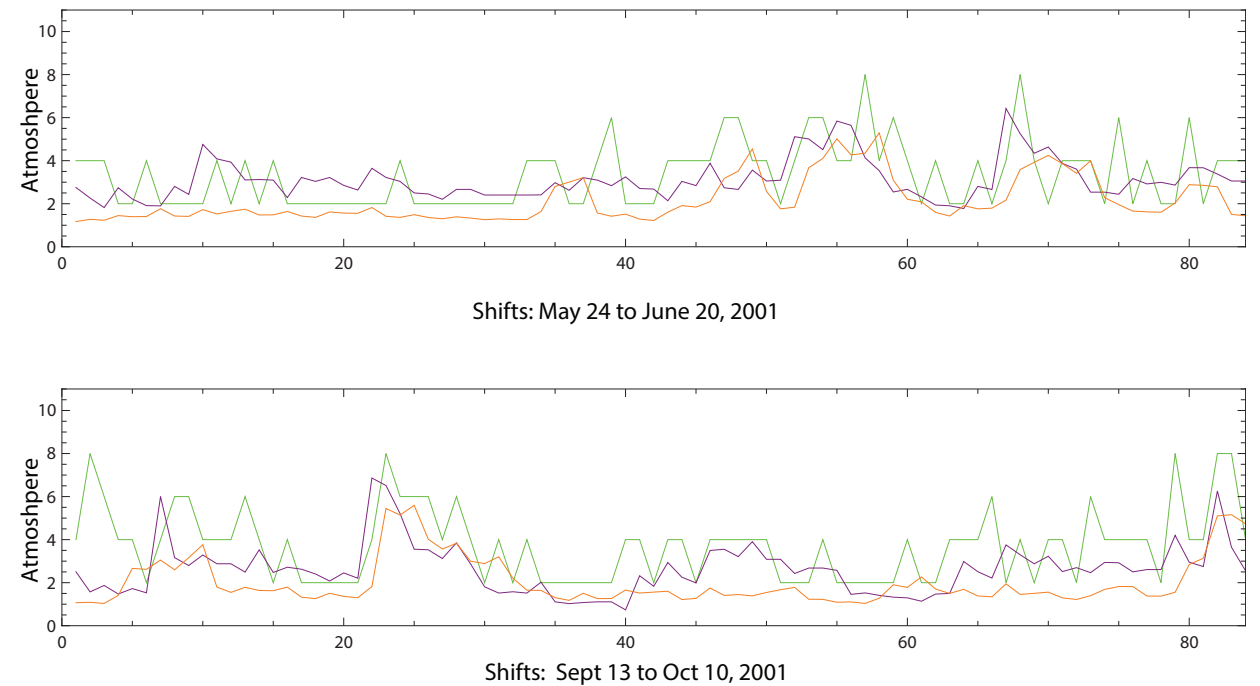

Figure 9: Predicted vs experimental values for the atmosphere on the two case periods. In both figures: experimental (green); kinetic model (orange), ANN (purple).

The corresponding deviations $S d / S v$ and $S d / N$ for the kinetic model and the ANN model are shown in Figs. 10.

Finally, the Pearson's and the Spearman's $\rho$ correlation coefficients are contrasted in Figs. 11.

By comparing the results of the kinetic and ANN approach on the two case periods (with the second period chosen as validation), it appears that the ANN predictions are somewhat closer to the experimental values, a result certainly achieved with less mathematical effort. However, the mean deviations and the analysis of the statistical coefficients over a span of multiple years suggest the kinetic model to be more reliable in the long run, i.e., its 

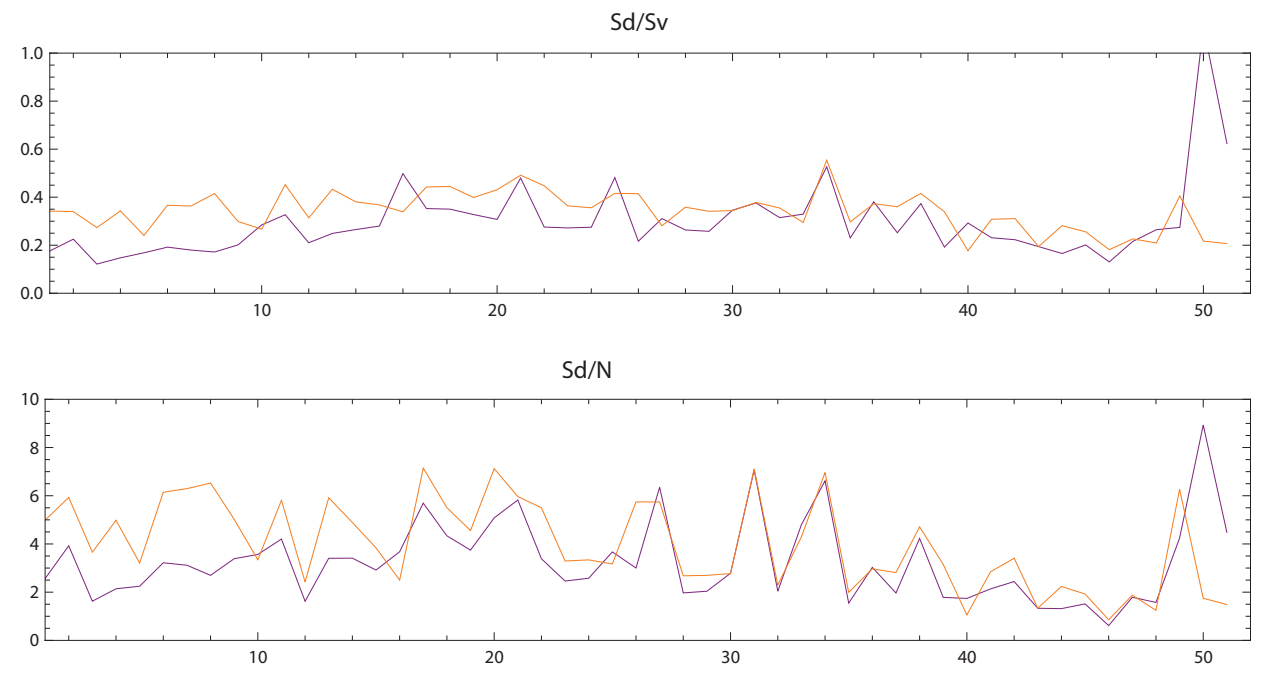

Figure 10: $S d / S v$ (top) and $S d / N$ (bottom) for the kinetic model (orange) and ANN (purple).
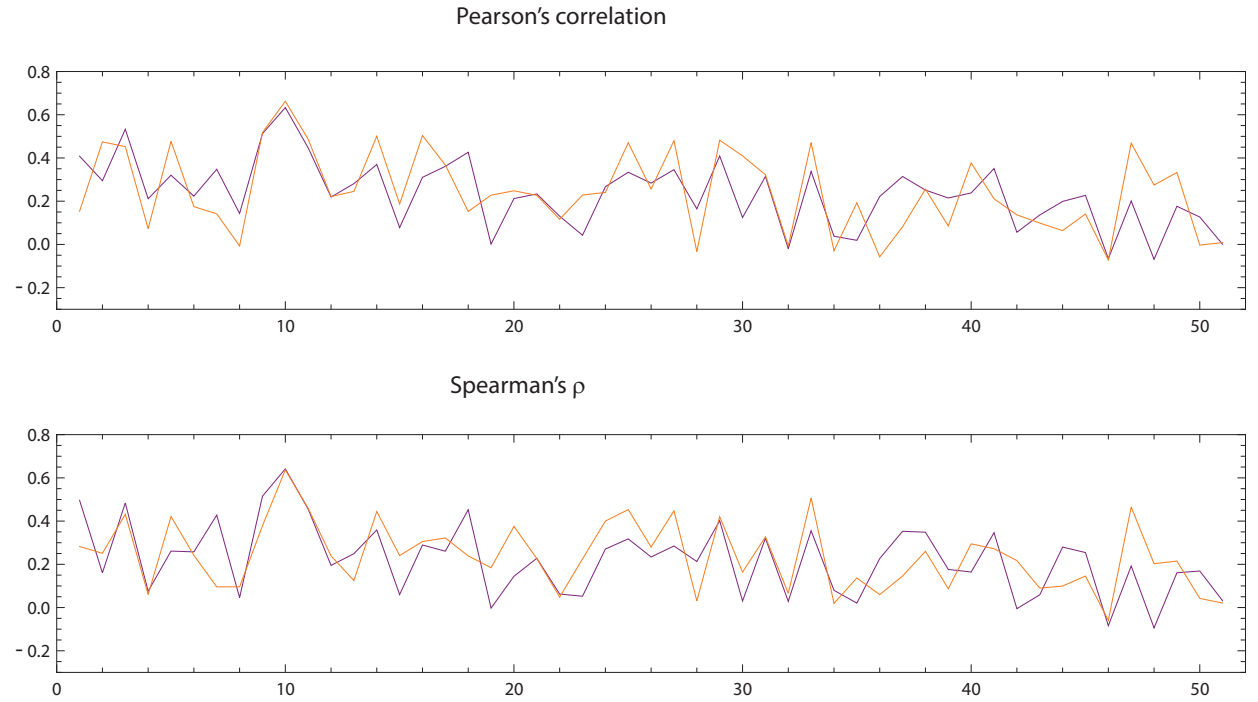

Figure 11: Pearsons's (top) and Spearman's $\rho$ correlation coefficient (bottom) for the kinetic model (orange) and ANN (purple).

predictions can be considered as acceptable even on periods that are quite far away from the two case periods over which the many parameters of the model had been optimized. Obviously, the assumption that the model parameters in the kinetic approach, as well as the trained ANNs, should provide meaningful predictions over extended periods of time, such as several months or even years, is questionable. As a matter of fact, within the extended period January 2001 - December 2004 several phases have been identified in the system, when the medical service was characterized by distinct functioning patterns. These, in principle, might 
require different trained ANNs, or at least a more specific and detailed analysis. However, as in the case of the kinetic model, it turns out that the observed phases can be put in correspondence with overall organizational changes in the service, and are evident only on a very long time scale. For example, the poorer performance of the ANN as a predicting tool in the last several months of 2004, which had also been clearly observed in the kinetic model, could be explained and somewhat expected on the basis of the functioning pattern of the medical unit. It is indeed remarkable that the two approaches exhibit such striking similarities, both for the mean deviations and for the correlation coefficients.

Obviously both models present certain advantages with respect to one another, mathematically as well as from the point of view of the psychosocial investigation, which in our opinion make them both worth pursuing further. On one hand, the kinetic approach has allowed a separate investigation of the climate of the two populations even in the absence of separate measurements/experimental data for the corresponding atmospheres. Importantly, this accounts for the somewhat different nature of the corresponding climate, which for patients is related to their well-being and behavior, while for the medical staff it is mostly expressed in terms of the stress they are subject to in performing their tasks. Moreover, the kinetic model seems to be able to provide more reliable long-term predictions. On the other hand, by conceptualizing the service as a whole, and reducing the number of measurements one needs to account for, an ANN approach is in a sense more "economical", both mathematically and from the point of view of the psychosocial analysis, and it is certainly easier to be implemented and used by a staff of non-mathematicians in the unit, as a (short-term) on-site predictive tool. A more in-depth comparison between different ANNs and kinetic models is currently being pursued, and it will be the subject of a future publication.

\section{Concluding remarks}

The major outcome of this work is a trained artificial neural network that can be used as a descriptive and predictive tool (possibly even by properly trained staff members in the medical ward) to help determine which parameters/events most deeply influence the quality of the ward, and which adjustments, on different time scales, may help improve the ward climate. What makes the present work particularly meaningful is that it relies on a large set of experimental data to which one can contrast and compare the predictions of the mathematical model, as well as the chance to develop strategies for improving the collection of new experimental data which can be inferred as a result of this work. For example, the collection of all input data utilized in this model (as listed in Table 2) on every shift, instead of once a day, as well as recording the atmosphere felt by patients and staff as separate measurements, might improve the performance of the network. Also, a detailed analysis of the statistical weights associated with the ANN implemented and tested in this model might be effective in suggesting which inputs, playing a minor role in the system dynamics, can be ignored without much loss. As a matter of fact, the data collection is still ongoing, and the previous mathematical investigation proposed by the authors has suggested refinements of the data collection process, which have been since then put in place in the unit, and which 
we plan to use in future developments of this study. Finally, a possible improvement of the model might rely on a more sophisticated algorithm for the reconstruction of the atmosphere based on the likeliness output of the various classes.

\section{Acknowledgments}

MLS is grateful to UCCS for the kind hospitality. BP acknowledges partial support for this work by the National Science Foundation under Grant No. DMS-1311883, and by the UCCS Graduate School and College of LAS.

1. J. Abbot, J. Marohasy, Application of artificial neural networks to rainfall forecasting in Queensland, Australia, Adv. Atm. Sci. 29 (2012) 717-730.

2. G. Ajmone Marsan, N. Bellomo, M. Egidi, Towards a mathematical theory of complex socio-economical systems by functional subsystems representation, Kin. Rel. Mod. 1 (2008) 249-78.

3. J.S. Allison, J. Heo, A.E. Iskandrian, Artificial neural network modeling of stress singlephoton emission computed tomographic imaging for detecting extensive coronary artery disease, Am. J. Cardiol. 95 (2005) 178-181.

4. O. Yu. Atkov, S. G. Gorokhova, A. G. Sboev, E. V. Generozov, E. V. Muraseyeva, S. Y. Moroshkinad, N. N. Cherniyc, Coronary heart disease diagnosis by artificial neural networks including genetic polymorphisms and clinical parameters, J. Cardiol. 59 (2012) 190-194.

5. P. Azimi, H. Reza Mohammadi, E. C Benzel, S. Shahzadi, S. Azhari, A. Montazeri, Artificial neural networks in neurosurgery, J. Neurol. Neurosurg. Psychiatry 0 (2014) $1-6$.

6. W.G. Baxt, Application of artificial neural networks to clinical medicine, The Lancet 346 (1995) 1135-1138.

7. S. Belciug, F. Gorunescu, Error-correction learning for artificial neural networks using the Bayesian paradigm. Application to automated medical diagnosis, J. Biomed. Inform. 52 (2014) 329-37.

8. N. Bellomo, A. Bellouquid, J. Nieto, J. Soler, Complexity and mathematical tools toward the modelling of multicellular growing systems, Math. Comp. Mod. 51 (2010) 441-451.

9. N. Bellomo, A. Bellouquid, J. Nieto, J. Soler, Multiscale biological tissue models and flux-limited chemotaxis for multicellular growing systems, Math. Mod. Met. Appl. Sci. 20 (2010) 1179-1207.

10. N. Bellomo, Modeling the hiding-learning dynamics in large living system, Appl. Math. Lett. 23 (2010) 907-911.

11. N. Bellomo, M. Lo Schiavo, Lectures Notes on the Generalized Boltzmann Equation, World Sci., London, Singapore, 2000.

12. N. Bellomo, D. Knopoff, J. Soler, On the difficult interplay between life, "complexity", and mathematical sciences, Math. Mod. Met. Appl. Sci. 23 (2013) 1861-1913.

13. A. Bellouquid, M. Delitala, Mathematical methods and tools of kinetic theory towards modelling complex biological systems, Math. Mod. Meth. Appl. Sci. 15 (2005) 1639-1666. 
14. M.L. Bertotti, M. Delitala, On a discrete generalized kinetic approach for modelling persuaders influence in opinion formation processes, Math. Comp. Model. 48 (2008) 1107-21.

15. P.J. Braspenning, F. Thuijsman, A. J. M. M. Weijters, Artificial Neural Networks: an Introduction to ANN Theory and Practice, Springer-Verlag, Berlin Heidelberg (1995).

16. S. Cao and J. Cao, Forecast of solar irradiance using recurrent neural networks combined with wavelet analysis, App. Therm. Eng. 25 (2005) 161-172.

17. C. Cercignani, The Boltzmann Equation and Its Application, Springer, New York, 1988.

18. S. De Lillo, M. Delitala, M.C. Salvatori Modelling epidemics and virus mutations by methods of the mathematical kinetic theory for active particles, Math. Mod. Met. Appl. Sc. 19 (2009) 1405-1425.

19. A. Dutt-Mazumder, C. Button, A. Robins, R. Bartlett, Neural network modelling and dynamical system theory: are they relevant to study the governing dynamics of association football players?, Sports Med. 41 (2011) 1003-17.

20. G.B. Fogel, Computational intelligence approaches for pattern discovery in biological systems, Brief. Bioinform. 9 (2008) 307-316.

21. S. Friis, Measurements of the perceived ward milieu: a reevaluation of the Ward Atmosphere Scale, Acta Psychiatr. Scand. 73 (1986) 589-599.

22. I. Gallo, A. Barra, P. Contucci, Parameter evaluation of a simple mean-field model of social interaction, Math. Mod. Meth. Appl. Sci. 19 (2009) 1427-1439.

23. M.W. Gardner, S.R. Dorling, Artificial neural networks: the multilayer perpectron: Review of Applic. in the Atmospheric Sciences, Atm. Env. 32 (1998) 2627-2636.

24. M. B. Gordon, J.-P. Nadal, D. Phan, V. Semeshenko, Discrete choices under social influence: Generic properties, Math. Mod. Meth. Appl. Sci. 19 (2009) 1441-1481.

25. I. Goryanin, O. Demin, Kinetic Modelling in Systems Biology, Chapman Hall/CRC, 2009.

26. M. M. Gouda, S. Danaher, C. P. Underwood, Application of artificial neural network for modelling the thermal dynamics of a buildings space and its heating system, Math. Comp. Mod. Dynam. Syst. 8 (2002) 333-344.

27. E. Jager, L. Segel, On the distribution of dominance in a population of interacting anonymous organisms, SIAM J. Appl. Math. 52 (1992) 1442-1468.

28. S.A. Kalogirou, Applications of artificial neural networks in energy systems. A review., Energy Convers. Manag. 40 (1999) 1073-1087.

29. J. Khan, J. S. Wei, M. Ringnèr, L. H. Saal, M. Ladany, F. Westermann, F. Berthold, M. Schwab, C. R. Antonescu, C. Peterson, P. S. Meltzer, Classification and diagnostic prediction of cancers using gene expression profiling and artificial neural networks, Nat. Med. 7 (2001) 673-679.

30. V.M. Krasnopolsky, Y. Lin, A neural network nonlinear multimodel ensemble to improve precipitation forecasts over continental US, Adv. Meteorology Volume 2012 (2012), Article ID 649450, 11 pages.

31. P.J. Lisboaa, A.F.G. Taktak, The use of artificial neural networks in decision support in cancer: A systematic review, Neural Netw. 19 (2006) 408-415.

32. M. Lo Schiavo, B. Prinari, A.V. Serio, Analysing quality with generalized kinetic methods, Math. Comp. Mod. 47 (2008) 1150-1166. 
33. M. Lo Schiavo, B. Prinari, A.V. Serio, Mathematical modeling of quality in a medical structure: a case study, Math. Comp. Mod. 54 (2011) 2087-2103.

34. M. Lo Schiavo, Population kinetic models for social dynamics: Dependence on structural parameters, Comput. Math. Appl. 44 (2002) 1129-1146.

35. M. Lo Schiavo, The modelling of political dynamics by generalized kinetic (Boltzmann) models, Math. Comp. Mod. 37 (2003) 261-281.

36. M. Lo Schiavo, Kinetic modelling and electoral competition, Math. Comp. Mod. 42 (2005) 1463-1486.

37. T. Manning, R.D Sleator, P. Walsh, Biologically inspired intelligent decision making. A commentary on the use of artificial neural networks in bioinformatics, Bioengineered 5 (2014) 1-16.

38. A. Mechaqrane, M. Zouak, A comparison of linear and neural network ARX models applied to a prediction of the indoor temperature of a building, Neur. Comp. Appl. 13 (2004) 32-37.

39. I. Melle, S. Friis, E. Hauff, T.K. Island, S. Lorentzen, P. Vaglum, The importance of ward atmosphere in inpatient treatment of schizophrenia on short-term units, Psychiatr. Serv. 47 (1996) 721-726.

40. T. Middelboe, T. Schjødt, K. Byrsting, A. Gjerris, Ward atmosphere in acute psychiatric in-patient care: patients' perceptions, ideals and satisfaction, Acta Psychiatr. Scand. 103 (2001) 212-219.

41. S. Moein, Medical Diagnosis Using Artificial Neural Networks, IGI-Global, Hershey, PA (2014).

42. R. Moos, Evaluating Treatment Environments. A Social Ecological Approach, John Wiley, New York, 1974.

43. C. Papaloukas, D.I. Fotiadis, A. Likas, L.K. Michalis, An ischemia detection method based on artificial neural networks, Artif. Intell. Med. 24 (2002) 167-178.

44. J.L. Patel, R.K. Goyal, Applications of artificial neural networks in medical science, Curr. Clin. Pharmacol. 2 (2007) 217-26.

45. P.J. Roebber, M.R. Butt, S.J. Reinke, T.J. Grafenauer, Real-Time Forecasting of Snowfall Using a Neural Network, Weather Forecast. 22 (2007) 676-684.

46. J.I. Rossberg, I. Melle, S. Opjordsmoen, S. Friis, The relationship between staff members working conditions and patients perceptions of the treatment environment, Int. J. Soc. Psychiatry 54 (2008) 437-46.

47. J.I. Rossberg, S. Friis, Patients' and staff's perceptions of the psychiatric ward environment, Psychiatr. Serv. 55 (2004) 798-803.

48. F. Sato, Y. Shimada, F. M. Selaru, D. Shibata, M. Maeda, G. Watanabe, Y. Mori, S.A. Stass, M. Imamura, S. J. Meltzer., Prediction of survival in patients with esophageal carcinoma using artificial neural networks, Cancer 103 (2005) 1596-605.

49. B. Schneider, S. White, M.C. Paul, Linking service climate and customer perceptions of service quality: test of a causal model, J. App. Psychol. 83 (1998) 150-163.

50. H. Tuvesson, C. Wann-Hansson, M. Eklund, The ward atmosphere important for the psychosocial work environment of nursing staff in psychiatric in-patient care, BMC Nursing 10 (2011) 12 .

51. R. Tagiuri, G.H. Litwin, Organizational Climate: Exploration of a Concept, Harvard 
University Press, Boston, 1968.

52. K. A. Urbanoski, B. H. Mulsant, G. Novotna, S. Ehtesham, B. R. Rush, Does the redesign of a psychiatric inpatient unit change the treatment process and outcomes?, Psychiatr. Serv. 64 (2013) 804-807.

53. World Health Organisation, Expert committee on mental health, (1953) Third report (Technical report series no 73) Geneva: WHO. 